\title{
GEOCHRONOLOGY AS A FRAMEWORK FOR INNER SOLAR SYSTEM HISTORY AND EVOLUTION
}

A White Paper for the Planetary Science Decadal Survey

July 6, 2020

Barbara A. Cohen, NASA Goddard Space Flight Center Greenbelt, MD, USA

(barbara.a.cohen@nasa.gov|301-614-6803)

Nicolle Zellner, Albion College

Meenakshi Wadhwa, Arizona State University

Brent D. Turrin, Rutgers University

Marissa M. Tremblay*, Purdue University

Timothy D. Swindle, Lunar and Planetary Laboratory, University of Arizona

Stephanie E. Suarez*, University of Houston

David L. Shuster, University of California

Justin I. Simon, NASA Johnson Space Center

Kirby D. Runyon*, Johns Hopkins Applied Physics Laboratory

Stuart J. Robbins*, Southwest Research Institute

Clive R. Neal, University of Notre Dame

Daniel P. Moriarty*, Universities Space Research Association/NASA Goddard Space Flight Center

Cameron M. Mercer*, Catholic University of America/NASA Goddard Space Flight Center Tom Lapen, University of Houston

Michelle R. Kirchoff, Southwest Research Institute

Steven J. Jaret*, American Museum of Natural History

Kip V. Hodges, Arizona State University

Gregory F. Herzog, Rutgers University

George Gehrels, University of Arizona

Barbara Frasl*, Catholic University of America/NASA Goddard Space Flight Center

Caleb I. Fassett, NASA Marshall Space Flight Center

Daniel R. Dunlap*, USRA/LPI/NASA Johnson Space Flight Center

Regina DeWitt, East Carolina University

Natalie M. Curran*, Catholic University of America /NASA Goddard Space Flight Center

Carolyn Crow*, University of Colorado Boulder

Fanny Cattani*, Catholic University of America/NASA Goddard Space Flight Center

Julia A. Cartwright, University of Alabama

Marc W. Caffee, Purdue University

Ricardo Arevalo Jr., University of Maryland

F. Scott Anderson, Southwest Research Institute

*early-career scientists 


\section{Executive Summary}

Major advances in planetary science will be driven by determining absolute ages of geologic units on multiple bodies in the inner Solar System in the next decade. Absolute ages calibrate bodyspecific chronologies and create a framework for understanding Solar System formation, the effects of impact bombardment on life, and the evolution of planets and their interiors. We recommend that the Decadal Survey:

- Support Mars Sample Return to ensure the return to Earth of a set of carefully-chosen, well-documented samples for geochronology and other critical studies of Mars' evolution and past habitability.

- Prioritize sample-return missions from planetary bodies in the inner Solar System that would answer fundamental questions about the timing of major events in planetary history.

- Advocate for sustained funding for in situ dating instruments to continue to raise their technology readiness levels for future exploration of planetary surfaces.

- Include a Solar System chronology mission on the New Frontiers mission list, setting the science goals of such a mission but allowing flexibility in how those goals are accomplished.

- Reemphasize the importance of an investment strategy for laboratory instrumentation and curation sufficient to provide for both replacement of existing capacity and development of new capabilities.

- Recommend that NASA partner with NSF on their Decadal geochronology initiative to leverage NASA's investments in infrastructure supporting planetary geochronology.

\section{Introduction}

Geochronology, the determination of isotopic ages for geologic events, underpins virtually every inquiry into the formation and evolution of planets and our Solar System. Geochronology provides the intertwined threads of when, how long, and how intense. For example, when did planetary bodies in our Solar System accrete, melt, and differentiate, forming core, mantle, and crust? How intense was early bombardment of the inner Solar System? How long were planetary heat engines active? How long were eruptions on other planetary bodies occurring and what were their frequencies? When was Mars warm and wet? How long have surfaces been exposed to the space environment? Absolute and relative isotopic ages create the framework for interpreting geologic events on individual planets in the context of Solar System evolution: what was going on elsewhere in the Solar System when that happened?

Our existing understanding of inner Solar System chronology is anchored to the crater density and analogy with impact rates on the Moon, under the assumptions that the lunar cratering history is well-constrained, and that flux rates for Mercury, Venus, Mars, and Vesta can be derived from the lunar rate. However, the lunar cratering record is unconstrained prior to the apparent bombardment of 4 Gyr ago, and conveys a roughly billion-year uncertainty between 1 and $3 \mathrm{Ga}$. Since the Apollo and Luna missions, only incremental advancement has been made in constraining the absolute ages for particular events that may help relate their histories to one another, defining epochs that seem to have little relation to each other (Fig 1). Compounding the uncertainties of the lunar cratering rate are the uncertainties in the assumptions required to extrapolate this curve to the other planetary bodies. Calibration of body-specific chronologies will place planetary surface processes into a common age framework that will lead to major advances in understanding Solar System formation, the effects of impact bombardment on life, and the evolution of planets and their interiors. 


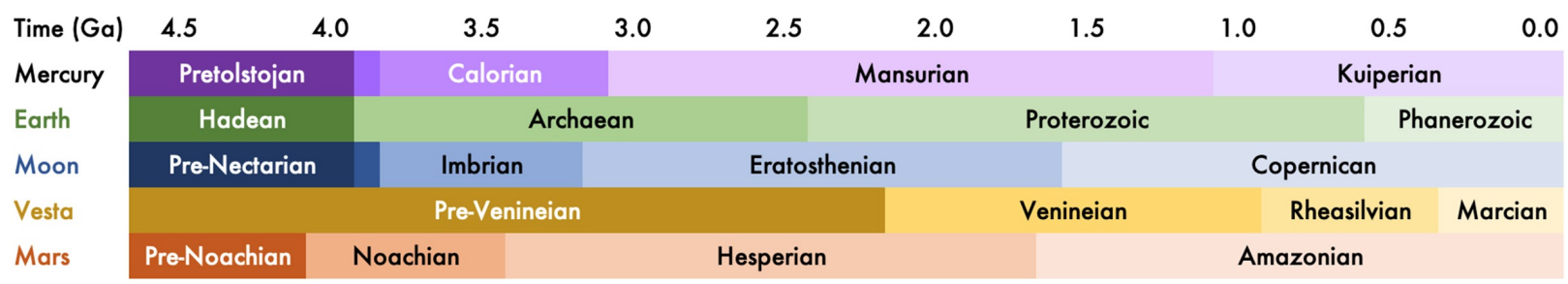

Solar System

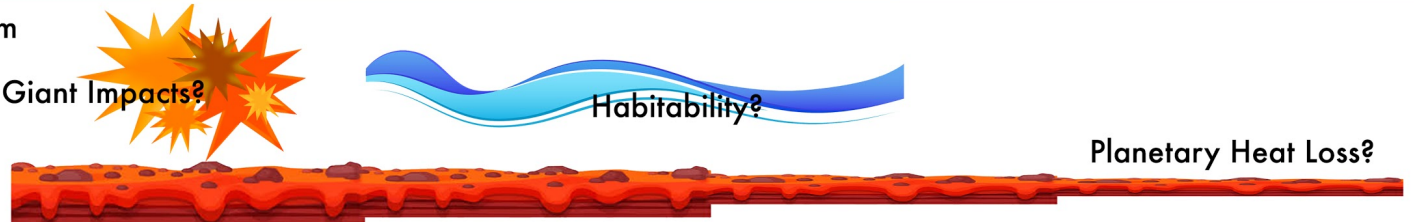

Fig. 1. Geologic ages on the inner planets, with best estimates of the absolute time of each. The current geologic age boundaries on one have little relationship to those on any other, making it impossible to interpret geologic evolution within a solar system context. Furthermore, the knowledge of the absolute ages of the boundaries is not as precise as the well-defined vertical lines would imply.

\section{Major Science Questions Driven by Geochronology}

Impacts and Solar System dynamics. Impact bombardment is one of the main geologic processes that affects all planetary bodies throughout the Solar System. The leading, but contentious, model for lunar impact history includes a pronounced increase in large impact events between around 3.9 $\mathrm{Ga}$, and possibly extending back in time to at least $4.2 \mathrm{Ga}$. Though recent work on lunar samples has supported the possibility that lunar samples were influenced by repeated sampling of Imbrium ejecta (Zellner, 2017), independent constraints come from impact ages of meteorites derived from asteroid parent bodies (e.g., Bogard, 2011; Marchi et al., 2013). Such a bombardment would have occurred on an Earth, and perhaps Mars, that had atmospheres, oceans, and continents, and may have influenced the course of biologic evolution. The dynamical models that enable an early bombardment are strongly linked to the processes describing the endgame of planet formation (reviewed in Bottke and Norman, 2017). These models describe the gas-dust dynamics of forming disks and giant planet migration that now may be invoked to understand our Solar System as well as systems of exoplanets around other stars. As we seek to better understand these other systems, we begin by asking a fundamental question: Is the Solar System typical or anomalous? One of the best ways to address this question is to determine what processes occurred and their timing and duration in the early Solar System and compare the results to what is seen in planetary systems that are currently forming around other stars. A key test of these dynamical models is whether the terrestrial planets and asteroid belt experienced a relative "lull" in impacts between formation and later bombardment. One way that this objective could be addressed is by establishing the formation time of large planetary basins by dating their impact-melt sheets. Important basins occur throughout the inner planets: South Pole-Aitken, Crisium, Nectaris on the Moon, Isidis and Argyre basins where impactite deposits are well preserved on Mars, Caloris on Mercury, Occator on Ceres, and Rheasilvia on Vesta. Obtaining absolute formation ages for these basins would place them either within the canonical cataclysm $(3.9 \mathrm{Ga})$ or as part of a declining bombardment in which most impact basins are older. 
Igneous evolution and interior processes. Ages for magmatic products provide strong constraints on the dynamics of magma oceans and crustal formation, the longevity and evolution of interior heat engines and mantle/crustal source regions. Whether a planet forms a rigid-lid and cools relatively slowly; cools more quickly by advective heat loss and magmatism; and/or this primordial lid founders and is recycled, are critical to the formation of planetary surfaces stable over periods lasting long enough for life to get a foothold. Moreover, these processes dictate whether these modes of planetary evolution and heat loss persist or evolve into the processes of plate tectonics that exist today on Earth. Our understanding of the thermal and compositional evolution of these bodies is limited by the lack of returned samples from known locations, and the absence of new constraints on their composition and absolute ages. On Mars and the Moon, the period from $\sim 1$ to $3 \mathrm{Ga}$ includes the cessation of abundant volcanism; a revised chronology could allow another billion years of volcanism and would overturn current models for planetary thermal evolution. Critical advances in understanding planetary volcanism would come from the geochronologic determination of the youngest and oldest basalts on each body along with ages for benchmarking igneous provinces such as Syrtis Major on Mars and irregular mare patches on the Moon.

Habitability. Our incomplete knowledge of absolute geochronology limits our ability to understand the timing of evolutionary milestones. The Curiosity mission demonstrated that aqueous habitable settings existed on Mars in surface environments well into the Hesperian and maybe later (Martin et al., 2017), yet most models for the evolution of martian climate suggest conditions were not likely favorable for surface habitable environments in much of the Hesperian, much less into the Amazonian. More broadly, we do not know whether the Noachian-Hesperian boundary, where a clement Mars became more acidic, occurred before, after or concurrent with the late heavy bombardment on the Moon and the oldest intact rocks on Earth, so martian climate change cannot yet be put into the context of Solar System history (Ehlmann et al., 2016). Knowing the absolute ages of the major martian epochs is critical to understanding how the habitability of the planets in our Solar System evolved over time.

\section{Strategies for 2023-2033}

We advocate a multi-pronged approach to weaving geochronology throughout the planetary missions and supporting research in the next decade (and beyond) that includes sample return, in situ dating, and laboratory analyses.

Sample Return. High-precision geochronological investigations of samples returned from selected locations on the Moon, including the Vision and Voyages New Frontiers target South Pole-Aitken Basin, would significantly advance our understanding of lunar chronology and Solar-System processes. As the oldest stratigraphically recognizable basin on the Moon, the South Pole-Aitken Basin (SPA) is a critical calibration point for relative geochronology of the Moon and other planets. An absolute age of SPA would anchor the impactor flux curve relatively early in planetary history. Additional constraints on both the old and young ends of the crater flux curve and magmatic history are also necessary, and numerous locations on the Moon, Mars, and asteroids carry compelling geochronology objectives (e.g., Jawin et al., 2019; Ryder et al., 1989, Beaty et al. 2019).

Though Mars sample return (MSR) efforts are largely driven by the search for astrobiologically relevant materials, a crucial objective for MSR is to establish absolute geochronological anchors 
for the impact history of Mars (McSween et al., 2020). The potential to collect samples suitable for these efforts was given high priority in Mars 2020 landing site and architecture discussions. Such samples would be amenable to multiple geochronological systems in state-of-the-art laboratories on Earth, as well as to other techniques (such as isotopic and trace element analysis) that illuminate the history of the planet. The selected Perseverance landing site, Jezero Crater, contains an extensive, well-cratered unit overlying the basin fill material, embaying the delta outcrops, though the origin of the unit as volcanic or sedimentary is not yet established (e.g., Goudge et al., 2018; Rogers et al., 2018). Investigations with the rover payload will determine if this unit is volcanic, volcaniclastic, or sedimentary. Returned samples from the unit may determine its age - either a crystallization age that would be relatable to the martian crater curve, or the formation and exposure age of the detrital precursors. However, significant challenges remain to the interpretation of this unit as a) volcanic and b) not buried and exhumed, which would affect the crater retention age. The region surrounding Jezero Crater also includes several other wellcratered surfaces that could be accessible with current notional rover paths in an extended mission. Evaluation will begin early next year as Perseverance lands and investigates this unit in particular. With the addition of the Returned Sample Scientists to the Perseverance team, NASA has started developing requirements and procedures for selection of appropriate samples for chronology studies in samples to be returned from Mars.

Recommendation: Support the later stages of Mars Sample Return after Perseverance to ensure the return to Earth of carefully chosen, well-documented samples for geochronology and other critical studies.

Recommendation: Prioritize sample-return missions from the Moon and asteroids that would answer fundamental questions about the timing of major events in planetary history.

In situ Dating. The number of geochronologically significant geologic terrains across the inner Solar System far exceeds our ability to conduct sample return from all of them. Previous Decadal Surveys concluded that sample return was then the only way to obtain reliable and interpretable geochronology. Today, however, in the absence of sample return, planetary scientists routinely use in situ geochemical (e.g., APXS, ChemCam) and isotopic techniques (e.g., mass spectrometers) to make measurements that although less precise than those made in terrestrial laboratories, are sufficient to resolve major questions. Recognizing the practical limits on sample return, over the last two decades, NASA has invested in the development of in situ dating techniques; through the MatISSE and DALI programs, the technology readiness levels of instruments using complementary radiogenic isotopic systems (K-Ar and Rb-Sr) are on track to become TRL 6 by 2023, when this Decadal Survey's priorities are implemented (reviewed in Cohen et al., 2019). These investments have made possible New Frontiers-class missions that would carry multiple, complementary instruments to conduct in situ dating with the precision needed to meet community-identified science goals (Cohen et al., 2020). Well-formulated questions might also be addressed using smaller payloads (e.g., single method of isotopic dating, downsized characterization suite) in, e.g., Discovery-class missions. Additionally, new remote-sensing work, geologic mapping, and site evaluation efforts have expanded the locations where safe landing sites allow access to lithologies of interest. We urge the Decadal Survey panel to include missions in the New Frontiers list that address compelling problems in geochronology by either sample return or in situ dating. 
Recommendation: Advocate for sustained funding to raise the technology readiness levels of instruments and to develop new instruments that enable the use of multiple complementary isotopic dating systems to ensure a robust range of choices for implementation.

Recommendation: Include a Solar System Chronology mission as one of the priorities on the New Frontiers mission list, setting the science goals of such a mission but allowing flexibility in how those goals are accomplished (e.g., sample return or in situ dating, choice of target body) to take advantage of both recent advances in science and creative implementation solutions that may emerge from the planetary science community.

Laboratory Facilities. Earth-based laboratory capabilities for returned samples must continue to advance in sensitivity, accuracy and precision, as well as efficiency in the handling and processing of diverse samples. In fact, the limited nature of sample-return missions like Stardust, Genesis, and Hayabusa drove the advancement of laboratory capabilities for the analysis of smaller and smaller samples and the streamlining of analytical protocols, now regular practice in laboratory geochronology (e.g., Stroud et al., 2020; Harrison, 2009; Shearer and Borg, 2006). In the coming decade, the OSIRIS-REx and Hayabusa2 missions will return samples of primitive, organic-rich asteroids, and JAXA's Martian Moons eXplorer (MMX) will return samples of the martian moon Phobos. The next decades hold the promise of sample return from the Moon, comets (e.g. Milam et al., 2020), and the surface of Mars. Collectively, these missions promise to revolutionize understanding of the origin and evolution of the Solar System and extend our framework of Solar System geochronology. For sample geochronology, the primary instruments are high-precision mass spectrometers, equipped with thermal or plasma ionization sources, secondary ion and noble gas mass spectrometers, and accelerator mass spectrometers. However, our current laboratory infrastructure is inadequate to meet the analytical challenges associated with unlocking the full potential held by extraterrestrial sample analysis (National Academies of Sciences and Medicine, 2019). Sustained investment in laboratory upgrades and advancements, as well as in training future generations of research analysts, will be needed to extract maximum scientific return from geochronological investigations of existing and future samples from planetary targets.

Recommendation: Emphasize to NASA that they should adopt the investment strategies recommended in the NASEM report. In particular, place high priority on investment in analytical instrumentation (including purchase, maintenance, technical oversight, and development) and curation (facilities and protocols) sufficient to provide for both replacement of existing capacity and development of new capabilities.

Opportunities to Partner. The Earth Science community has long been the home for geochronology, driving instrumentation, techniques, and fundamental knowledge. Every significant advance in geochronology has produced a paradigm-shifting breakthrough in our understanding of Earth's history. Without quantitative knowledge of absolute and relative time, no modern discipline with a historical focus could function (Harrison et al., 2015). The most recent report from the National Science Foundation (NSF) Division of Earth Sciences (EAR) decadal survey (National Academies of Sciences and Medicine, 2020) recommends a National Consortium for Geochronology, stating that "Given that nearly all the high-priority science questions require improved constraints on the ages and rates of geologic processes, it will be important for EAR to build enhanced geochronologic capabilities." Planetary geochronology benefits from close 
integration with the terrestrial community to infuse best practices and encompass not just the Planet Earth but all the planets in our Solar System.

Recommendation: Recommend that NASA partner with NSF on their geochronology initiative to leverage NASA's investments in infrastructure and funding that supports planetary geochronology.

\section{References}

Beaty D. W., et al. (2019) The potential science and engineering value of samples delivered to Earth by Mars sample return. Meteoritics \& Planetary Science 54, S3-S152.

Bogard D. D. (2011) K-Ar ages of meteorites: Clues to parent-body thermal histories. Chemie der Erde 71, 207-226.

Bottke W.F. and Norman M.D. (2017) The Late Heavy Bombardment. Annual Review of Earth and Planetary Sciences 45, 619-647.

Cohen B. A. et al. (2020) Geochronology for the Next Decade: A Planetary Mission Concept Study.

Cohen B. A., Malespin C. A., Farley K. A., Martin P. E., Cho Y., and Mahaffy P. R. (2019) In Situ Geochronology on Mars and the Development of Future Instrumentation. Astrobiology 19, 1303-1314.

Ehlmann B. L., et al. (2016) The sustainability of habitability on terrestrial planets: Insights, questions, and needed measurements from Mars for understanding the evolution of Earth-like worlds. Journal of Geophysical Research: Planets 121, 1927-1961.

Goudge T. A., Mohrig D., Cardenas B. T., Hughes C. M., and Fassett C. I. (2018) Stratigraphy and paleohydrology of delta channel deposits, Jezero crater, Mars. Icarus 301, 58-75.Harrison M., Baldwin S., Caffee M., Gehrels G., Schoene B., Shuster D., and Singer B. (2015) Geochronology: It's about time. Eos Trans. AGU 96.

Harrison T. M. (2009) The Hadean Crust: Evidence from $>4$ Ga Zircons. Annual Review of Earth and Planetary Sciences 37, 479-505.

Jawin E. R., Valencia S. N., Watkins R. N., Crowell J. M., Neal C. R., and Schmidt G. (2019) Lunar Science for Landed Missions Workshop Findings Report. Earth and Space Science 6, 240 .

Marchi S., Bottke W. F., Cohen B. A., Wünnemann K., Kring D. A., McSween H. Y., Sanctis M. C. D., O’Brien D. P., Schenk P., Raymond C. A., and Russell C. T. (2013) High-velocity collisions from the lunar cataclysm recorded in asteroidal meteorites. Nature Geoscience 6, 303307.

Martin P. E., Farley K. A., Baker M. B., Malespin C. A., Schwenzer S. P., Cohen B. A., Mahaffy P. R., McAdam A. C., Ming D. W., Vasconcelos P. M., and Navarro-González R. (2017) A TwoStep K-Ar Experiment on Mars: Dating the Diagenetic Formation of Jarosite from Amazonian Groundwaters. Journal of Geophysical Research 122, 2803-2818.

McSween H. Y., Grady M. M., McKeegan K., Beaty D. W., and Carrier B. L. (2020) Why Mars Sample Return is a Mission Campaign of Compelling Importance to Planetary Science and Exploration. A White Paper for the Planetary Decadal Survey. 
Milam, S.N., and others (2020) Volatile Sample Return in the Solar System. A White Paper for the Planetary Decadal Survey.

National Academies of Sciences E. and Medicine (2019) Strategic Investments in Instrumentation and Facilities for Extraterrestrial Sample Curation and Analysis. The National Academies Press, Washington, DC.

National Academies of Sciences E. and Medicine (2020) A Vision for NSF Earth Sciences 20202030: Earth in Time. The National Academies Press, Washington, DC.

Rogers A. D., Warner N. H., Golombek M. P., Head Iii J. W., and Cowart J. C. (2018) Areally Extensive Surface Bedrock Exposures on Mars: Many Are Clastic Rocks, Not Lavas. Geophysical Research Letters 45, 1767-1777.Ryder G., Spudis P. D., and Taylor G. J. (1989) The case for planetary sample return missions: Origin and evolution of the Moon and its environment. Eos, Transactions American Geophysical Union 70, 1495-1509.

Shearer C. and Borg L. (2006) Big returns on small samples: Lessons learned from the analysis of small lunar samples and implications for the future scientific exploration of the Moon. Chemie der Erde 66, 163-185.

Stroud, R. M., and others (2020) Development of Capabilities and Instrumentation for Curation and Analysis of Returned Samples White paper for the Planetary Decadal Survey.

Zellner N. E. B. (2017) Cataclysm no more: New views on the timing and delivery of lunar impactors. Origins of Life and Evolution of Biospheres 47, 261-280. 